- Introduces the reader to a safe multiple agent IV sedation technique.

- Enables an increased understanding of the use of IV sedation in children.

- Removes the myth that IV sedation is a step lighter than general anaesthesia.

- Introduces the concept of IV sedation being as light or as deep as desired, depending on dosage.

\title{
Intravenous conscious sedation in children for outpatient dentistry
}

\author{
M. S. Mikhael, ${ }^{1}$ S. Wray ${ }^{2}$ and N. D. Robb ${ }^{3}$
}

The use of general anaesthesia for dental treatment in the NHS outside hospitals has changed over time. Although deaths are uncommon during or immediately after general anaesthesia for dental treatment, they are more likely to occur than with other methods of pain and anxiety reduction, such as local anaesthesia and conscious sedation. Inquiries into recent anaesthetic deaths in dental practice have been critical of the standard of care provided in areas such as pre-operative assessment, monitoring, resuscitation and transfer to specialist critical care facilities. ${ }^{1}$

In 1990 a report was produced which made over 50 recommendations aimed at reducing the risk of death or adverse events during dental treatment, including procedures under general anaesthesia. $^{2}$ The recommendations were implemented in the NHS through the Regional Health Authorities, but their implementation was neither comprehensive nor consistent.

In November 1998, the General Dental Council (GDC) acted to strengthen these recommendations by introducing guidance related to the provision of general anaesthesia for dental treatment. ${ }^{3}$ After 1998, the use of general anaesthesia for dental procedures outside of a hospital setting reduced dramatically, and since

*Consultant Anaesthetist, Barking, Havering and Redbridge Hospitals Associate University NHS Trust; ${ }^{2}$ Specialist Registrar in Anaesthesia, Barts and The London School of Anaesthesia, ${ }^{3}$ Senior Lecturer in Sedation in Relation to Dentistry, Glasgow Dental Hospital and School

Correspondence to: Dr Magdi Mikhae

Email:magdimikhael@btinternet.com

\section{Refereed Paper}

Accepted 16 November 2006

DOI: $10.1038 /$ bdj.2007.835

${ }^{\circ}$ British Dental Journal 2007; 203: 323-331
January 2002, general anaesthesia for dental treatment can only be carried out in hospitals with a critical care facility. ${ }^{1}$ These changes have been accompanied by an increase in the use of sedation techniques as an alternative to general anaesthesia, so as the use of conscious sedation increases, guidelines for its use need to be developed.

Using a combination of midazolam, alfentanil and ketamine in a fixed ratio of drug doses per kilogram of body weight in children aged three to ten years, for a range of outpatient dental procedures over a 21-month period (January 2000-September 2001), the following is observed.

'Conscious sedation', according to Implementing and ensuring safe sedation practice for healthcare procedures in adults, a report by an Intercollegiate Working Party organised by the Royal College of Anaesthetists, is a technique using drug(s) to produce depression of the central nervous system, allowing treatment to be carried out. Verbal contact must be maintained at all times and the $\operatorname{drug}(\mathrm{s})$ and technique used should have a safety margin wide enough to render loss of consciousness unlikely. If verbal contact is lost, the patient requires a level of care identical to that needed for general anaesthesia. ${ }^{4}$

Providing intravenous conscious sedation in children creates additional problems to its use in adults. For the majority of adult dental patients, the provision of sedation is sufficient to allow the dental surgeon to infiltrate local anaesthetic. Children require a combination of sedation, amnesia and analgesia. Single drugs therefore may be taken to limits of safety.

If psychotropic sedation is used alone (for example, midazolam), the child may not tolerate local anaesthetic injections, leading to the child crying and distressing the parents. If analgesia alone is given, the child will remain anxious and may be too uncooperative for the procedure to continue. A balanced technique may therefore provide analgesia so the child is able to tolerate the local anaesthetic injections and to provide sedation to allow the child to cooperate with the procedure and remain calm. The technique must be carefully titrated to effect, to prevent 'over-sedation' leading to loss 
of consciousness, loss of airway control and respiratory depression.

To achieve the requirements of intense analgesia and sedation, we used a combination of midazolam, alfentanil and ketamine. The use of a combination of agents also reduces the dose of each individual agent needed, so reducing the risk of potential side effects of each, although the sedative effects are clearly additive.

1. Midazolam has been widely used for the sedation of patients for minor procedures for a long time. It is painless on injection, has rapid onset, a short duration of action due to distribution, inactive metabolites and powerful amnesic properties. It may however cause respiratory depression, if used as a sole agent in large doses.

2. Alfentanil has rapid onset, a short duration of action, gives intense analgesia and has minimal cardiovascular effects, although it does cause some degree of respiratory depression. However, in higher doses it can cause bradycardia and hypotension, and when given in combination with midazolam, its half-life is increased due to metabolism by the same hepatic enzymes.

3. Ketamine has ideal properties to be used for the conscious sedation of paediatric dental patients. It provides intense analgesia and amnesia without respiratory depression or loss of laryngeal reflexes. The documented unpleasant side effects of ketamine, such as vivid dreams and emergence phenomena, are reported to be less common in paediatric patients and may be reduced by the concurrent use of benzodiazepines and opioids. ${ }^{5}$ Although more recent evidence suggests no benefit of adding benzodiazepines to the use of ketamine alone, ${ }^{5}$ benzodiazepines have been added to reduce the potential for vivid dreams and to reduce the dose of ketamine.

\section{The conduct of sedation}

On arrival in the dental surgery, all parents were given a questionnaire to complete in order to assess the health of their child. A medical history, history of previous surgery and anaesthetics, drug history and allergies were obtained. All children attending the dental lists who were ASA grade I or II were included in our report with no exclusions. Any child who is assessed as ASA grade III or IV is required to have their sedation for dental procedures undertaken in a hospital. ${ }^{6}$

Following assessment of the child, informed consent for the operation and the sedation technique was obtained from the parents. Possible complications including post-operative drowsiness, blurred vision, abnormal behaviour and nausea and vomiting were explained. The parents signed that they consented to the procedure, that they had been informed of the complications and that they had been issued with post-operative instructions. After signing the consent, the parents and child sat in the waiting area and a topical local anaesthetic gel was applied to the dorsum of the hands of the child if difficulty in tolerating cannulation was anticipated.

Sedation was carried out with the injection of ketamine $0.2-0.3 \mathrm{mg} / \mathrm{kg}$, alfentanil 5-20 $\mu \mathrm{g} / \mathrm{kg}$ and midazolam $0.1-0.2 \mathrm{mg} / \mathrm{kg}$ (Table 1). Following injection of the agents, the cannula was flushed with $5 \mathrm{ml}$ of $0.9 \%$ saline. These doses were chosen as clinical experience indicates that they provide the desired level of sedation consistent with the approved definition. The doses had been titrated to effect in patients at a time when general anaesthesia was in use in paediatric dental clinics, so the sedation could have been converted to general anaesthesia if required.

We started with the lower dose range and titrated the rest if necessary. Ninety seconds after the administration of the agents, the dentist assessed that the child was still maintaining verbal contact, and the ODP or dental nurse confirmed this. Local anaesthetic was injected as required by the dental treatment to be carried out. After a further five minutes, the dentist again assessed that verbal contact was maintained, and dental treatment commenced.

The dentist was requested to perform all the cavity preparations and extractions if scheduled during the initial period of sedation in order to allow less painful or distressing procedures to continue as the sedation became lighter. If the procedure exceeded 30 minutes duration or the child started to show signs of not tolerating the procedure, a further increment of alfentanil $5 \mu \mathrm{g} / \mathrm{kg}$ was administered, with a further increment of midazolam $75 \mu \mathrm{g} / \mathrm{kg}$ added three

\begin{tabular}{|l|l|}
\hline \multicolumn{2}{|l}{ Table 1 Sedation drugs } \\
\hline Drug & Dose per $\mathrm{kg}$ of body weight \\
\hline Ketamine & $0.2-0.3 \mathrm{mg} / \mathrm{kg}$ \\
\hline Alfentanil & $5-20 \mu \mathrm{g} / \mathrm{kg}$ \\
\hline Midazolam & $0.1-0.2 \mathrm{mg} / \mathrm{kg}$ \\
\hline
\end{tabular}

\begin{tabular}{|l|l|}
\hline \multicolumn{2}{|l|}{ Table 2 Sedation top-ups } \\
\hline Drug & Dose per $\mathrm{kg}$ of body weight \\
\hline Alfentanil & $5 \mu \mathrm{\mu g}$ \\
\hline$+/-$ & \\
\hline Midazolam & $0.1-0.2 \mathrm{mg} / \mathrm{kg}$ \\
\hline
\end{tabular}

Table 3 Agreed discharge policy

1. Walk unaided in a straight line

2. Answer questions sensibly and with clear speech

3. Pain free

4. Free from side effects (PONV, visual disturbance)

\begin{tabular}{l|l}
\multicolumn{2}{|c}{ Table 4 Verbal contact after sedation } \\
\hline $\begin{array}{l}\text { Verbal contact maintained } \\
\text { throughout }\end{array}$ & $994(99.4 \%)$ \\
\hline $\begin{array}{l}\text { Verbal contact not maintained } \\
\text { throughout }\end{array}$ & $6(0.6 \%)$ \\
\hline
\end{tabular}

minutes later if the desired effect was not achieved (Table 2).

The dentist assessed the child continually to ensure that verbal contact was maintained, and blood pressure was measured every five minutes throughout the procedure. Pulse oximetry was displayed and if at any time the haemoglobin oxygen saturation falls below $92 \%$, supplemental oxygen was administered via nasal cannula at a flow rate of two litres per minute.

Resuscitation equipment and emergency drugs are available at all times and protocols for anaphylaxis and cardiac arrest management were displayed in the operating room. The anaesthetist, dentists, ODPs and recovery nurses have all completed the Advanced Life Support Provider (ALS) course, and routinely 
received ALS updates in the surgery every two years.

At the end of the dental procedure, the child was transferred to the recovery room. Pulse oximetry and blood pressure monitoring continued. The child was supervised by the recovery nurse. The parents are allowed to see the child in recovery. The children walk assisted to a special seated area, to wait until they are fully alert.

The children were discharged from the clinic when they were able to walk unaided in a straight line, could answer questions sensibly with clear speech, and were free from pain or nausea and vomiting (Table 3). The sedationist reviewed all children before discharge. On discharge, a questionnaire was given to the parents to complete and return at their review visit. The parents were asked firstly how much their child could remember of the dental treatment, secondly, whether they were satisfied with the sedation and would consider it again for their children in future; thirdly, whether their child had suffered any untoward effects and finally, how long they felt their child had taken to assume completely normal behaviour after the procedure.

\section{Outcome and progression (Fig. 1)}

With this technique of conscious sedation the majority of cases (993 out of $1,000)$ were completed successfully.

Some children could not cope with intravenous cannulation. They would therefore be rescheduled for treatment as the procedure would be abandoned if the child could not tolerate indwelling cannula insertion, and/or parents may not consent to light restraint of the child for cannulation.

Occasionally some children would require inhalation sedation for cannulation and some may have local anaesthetic gel applied to the dorsum of the hand 20 to 30 minutes prior to cannulation, on the parents' request or if there was anticipated difficulty in the child tolerating cannulation following parental discussion. Most children tolerated cannulation without local analgesia.

of the children who had successful cannulation, all had sedation with ketamine, alfentanil and midazolam, followed by commencement of their dental work. After the administration of sedation, verbal contact was maintained

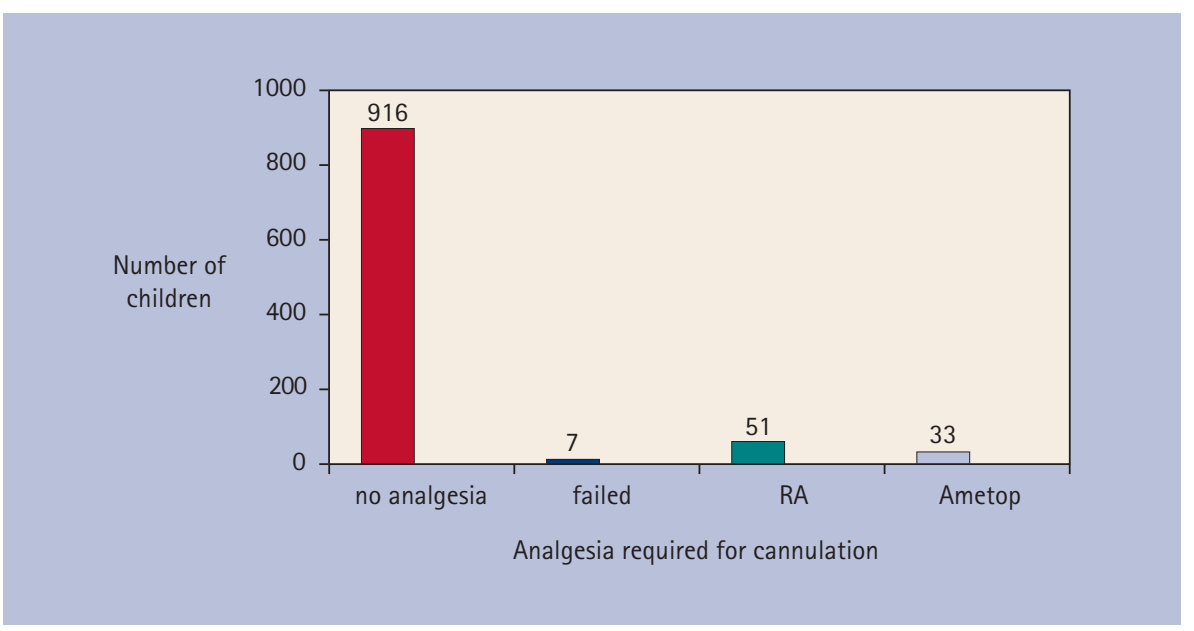

Fig. 1 Outcome of cannulation

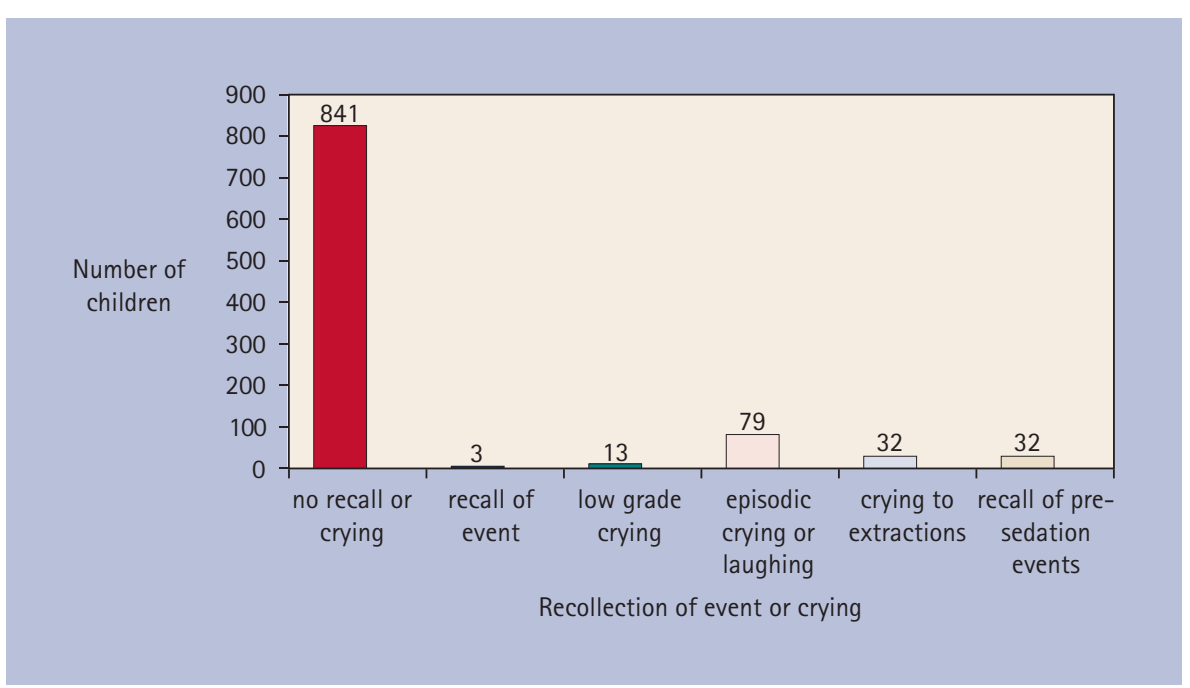

Fig. 2 Recall of post-cannulation events

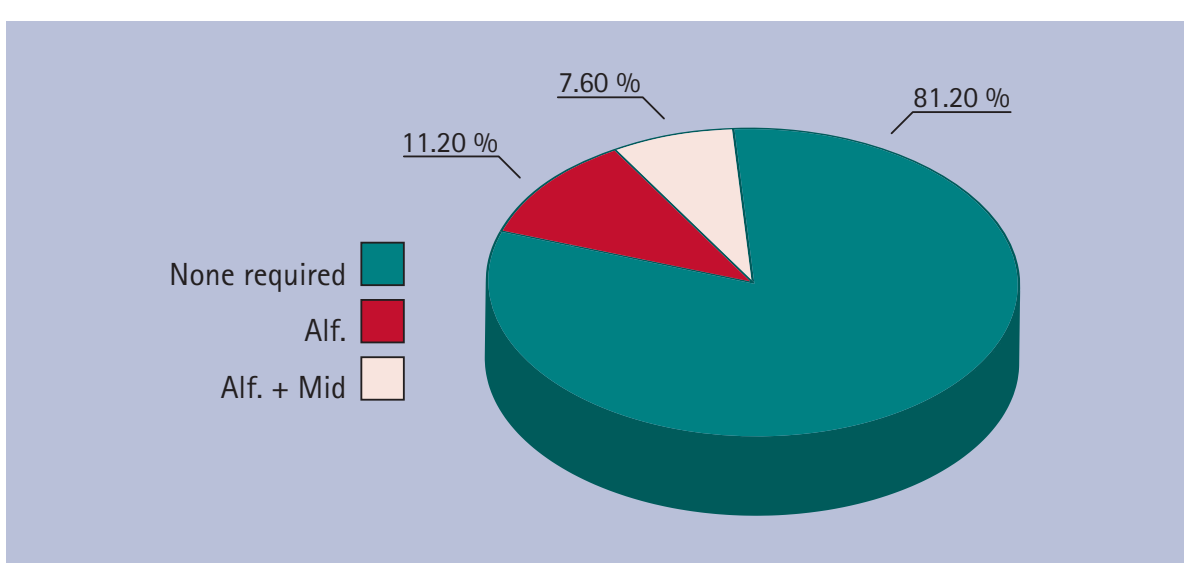

Fig. 3 Requirement for sedation top-ups

throughout in most of the children (994 out of 1,000 cases) (Table 4). They appeared not to be distressed by the local anaesthetic injection, but were readily responsive to questions or remarks made by the sedationist, dentist or assistants. Verbal contact was not maintained in six cases, however, they continued to look at specific targets within the room, had spontaneous movements and their airway was maintained. They generally had shorter procedures and the loss of verbal contact tended to occur after the termination of the surgical stimulus.

Recall of post-cannulation events (Fig. 2), for example administration of sedation, injection of local anaesthetic or the dental procedure itself occurred in three children (0.3\%). Thirteen (1.3\%) had continuous low grade crying throughout the 
procedure, not related to the dentistry. Several of them asked for their mother to be present during the procedure but they had no recall of events afterwards.

A minority of children (7.9\%) had one or two episodes of crying or laughing not related to the procedure, usually in the waiting period between activities. The children were asked at the time why they were laughing or crying but they could not provide a reason and they had no recall of this afterwards. Possible explanations for this are emergence dreams or visual hallucinations in relation to the administration of ketamine, or indeed the euphoric effect of ketamine for which it is commercially abused. Thirty-two children (3.2\%) cried specifically at the time of teeth extraction but did not recall these events in recovery. A further 32 patients (3.2\%), mostly older children, recalled perisedation conversations while they were in the recovery area but they were not distressed by these and had no recall of post-cannulation events.

Sedation top-ups were given as planned (Fig. 3). The percentage of cases receiving alfentanil plus midazolam administered three minutes later was $7.6 \%$ while $11.2 \%$ received a top up of alfentanil alone. No child received a second dose of ketamine.

The dental procedures ranged in duration from three to 67 minutes, with a mean time of 26 minutes. Surgery was planned not to exceed one hour.

The problems and side effects (Fig. 4) encountered with our technique of conscious sedation included hypoxia, postoperative nausea and vomiting (PONV), visual disturbances, lack of mouth sensation and in two cases, the recall of serious incidents from the distant past.

Hypoxia, defined as pulse oximeter readings lower than 92\% oxygen saturations, occurred in eight patients $(0.8 \%)$. It tended to be associated with insertion of the retractor into the mouth, particularly in children with large adenoids and tonsils, despite verbal contact being maintained. In all cases, hypoxia resolved spontaneously or with the administration of supplemental oxygen. PONV occurred in 53 patients (5.3\%), and anti-emetics were required to be given to 31 children (3.1\%).

Visual disturbances were a problem reported by 216 children $(21.6 \%)$ in the recovery period. Problems included

\section{Table 5 Parental response to questionnaire}

\begin{tabular}{|c|c|c|}
\hline 1. Did your child recall anything of the procedure after the needle? & $\begin{array}{l}\text { YES } \\
\text { NO }\end{array}$ & $\begin{array}{l}\% \\
7 \%\end{array}$ \\
\hline $\begin{array}{l}\text { 2. How long did your child take to assume completely normal behaviour } \\
\text { after administration of sedation? }\end{array}$ & $\begin{array}{l}1-2 \text { hours } \\
2-3 \text { hours } \\
3-4 \text { hours }\end{array}$ & $\begin{array}{l}79.4 \% \\
19.3 \% \\
1.3 \%\end{array}$ \\
\hline $\begin{array}{l}\text { 3. Are you satisfied with the sedation your child received for dental } \\
\text { treatment? }\end{array}$ & $\begin{array}{l}\text { YES } \\
\text { NO }\end{array}$ & $\begin{array}{l}99.3 \% \\
0.7 \%\end{array}$ \\
\hline 4. Would you consider sedation for your children & $\begin{array}{l}\text { YES } \\
\text { NO }\end{array}$ & $\begin{array}{l}99.8 \% \\
0.2 \%\end{array}$ \\
\hline
\end{tabular}

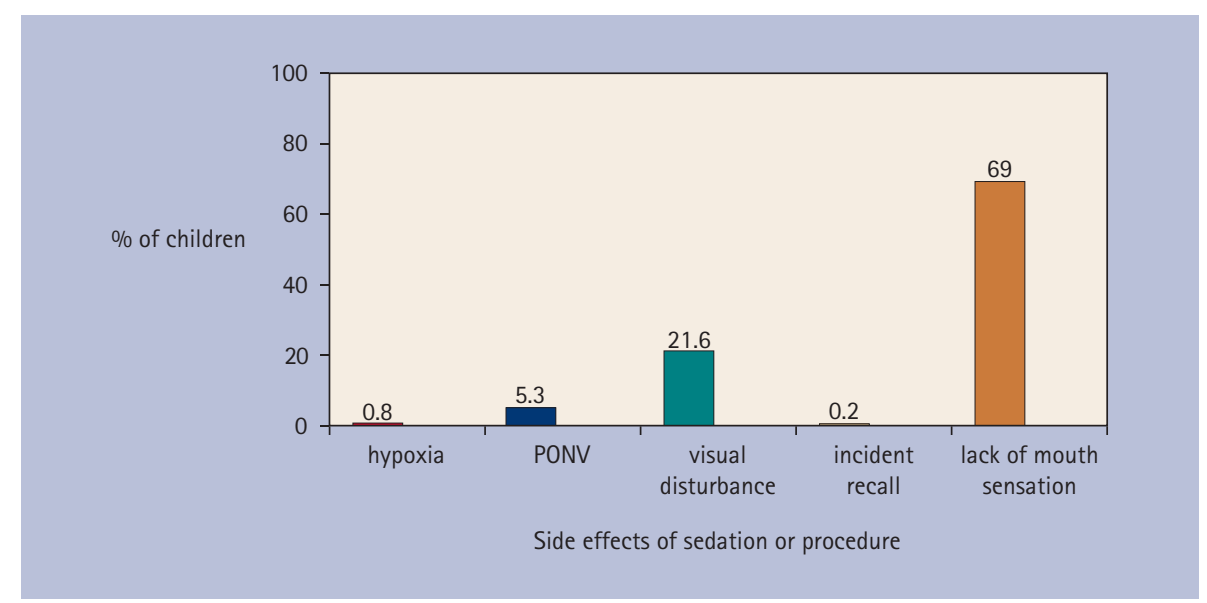

Fig. 4 Intra-operative and post-operative observed side effects

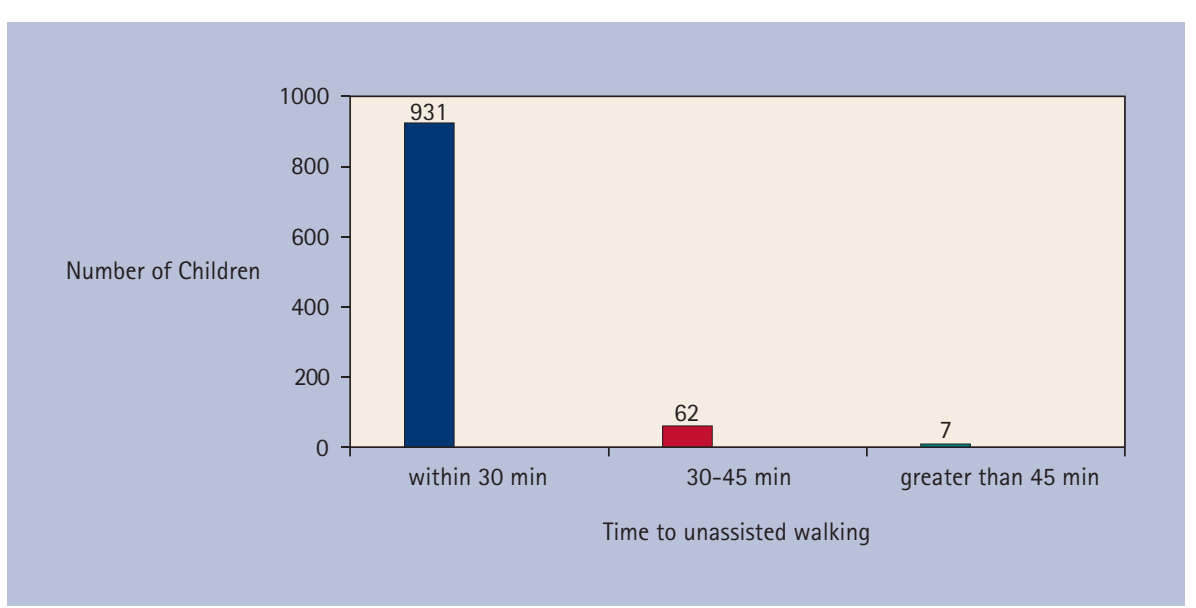

Fig. 5 Unassisted walking post-sedation

diplopia, visual hallucinations and vertical and horizontal nystagmus. In all children, visual disturbances had resolved by the time of discharge. Two children recalled serious incidents from their past while in the recovery. A seven-year-old girl had vivid recollections of a burglary three years previously at her home when she had been threatened by the burglar, and a 10-year-old girl recalled non-sexual abuse she had suffered from a male relative.

The most common side effect of the procedure reported by the children post-operatively was a lack of mouth sensation, which 690 children (69\%) complained of finding unpleasant to varying degrees. Interestingly, this figure correlates well with a dental audit at the surgery, where of patients who had dental procedures under local anaesthesia alone, 70\% complained of lack of mouth sensation post-operatively.

After the procedure, the children remained in the recovery area for 1015 minutes, supervised by a recovery nurse. When recovered sufficiently enough to walk, they were assisted to 
a special seated area to wait with their parents until satisfying the discharge criteria. After leaving recovery, over 93\% could walk unassisted in a straight line within 30 minutes, and over 99\% of children within 45 minutes (Fig. 5). The discharge criteria of answering questions sensibly with clear speech, being able to walk in a straight line and being free from side effects, were met by over 99\% of children within 60 minutes. Less than $1 \%$ of children had their discharge delayed for longer than 60 minutes. These children tended to have had shorter procedures or a change in the treatment plan; for example, surgery finishing shortly after a sedation top-up had been administered.

On discharge from the dental clinic, the parents were given a questionnaire to complete (Table 5). We received a $100 \%$ reply rate due to the practice of the dental surgeon who routinely held a review clinic when children were seen with their parents, during which visit the questionnaires were returned.

Although a subjective criteria, when asked how long they felt that their child had taken to assume completely normal behaviour after the sedation, 79.4\% of parents said their child was behaving normally within two hours, and 100\% within four hours. The parental satisfaction with the technique was very high (99.3\%); all of these patients and the children of the $0.7 \%$ of parents who expressed dissatisfaction later returned to the clinic for further dental procedures under conscious sedation. However, this may possibly be related to the relatively long waiting lists for the provision of primary dental care in hospitals following the cessation of general anaesthesia for dental procedures in dental surgeries. Overall, 99.8\% of parents stated that they would definitely consider conscious sedation again for their children in future.

Following the initial 1,000 cases we proceeded with random audit of 50 cases at any one time. We repeated the audit at regular intervals to assess whether results agreed with initial reports.

A further 5,000 cases were performed adhering to the original practice. The only difference was the introduction of titration of the mixture to replace the fixed dose regimen.

Results were almost identical except for the extremely small number of the deeply inadvertently sedated children was avoided by titration techniques.

\section{The clinical challenge}

Sedation is a continuum from the 'awake' state; it is a technique using pharmacological agents to reduce the level of consciousness and anxiety of the patient enough to allow them to tolerate an unpleasant procedure. The American Society of Anaesthesiologists (ASA) defines moderate or conscious sedation as a drug-induced depression of consciousness during which patients respond purposefully to verbal commands, either alone or accompanied by light tactile stimulation. No interventions are required to maintain a patent airway, and spontaneous ventilation is adequate. Deep sedation where patients cannot be easily roused but respond purposefully following painful stimulation and where assistance may be required to maintain a patent airway ${ }^{7}$ is considered to require the same level of care as general anaesthesia in the UK.

In our opinion, if you adhere strictly to the definition of conscious sedation in outpatient dentistry, then sedation is safer than general anaesthesia. However, if deep sedation is employed, the risks of airway obstruction, hypoventilation and hypoxia are greatly increased, with a higher associated mortality.

An ideal regimen of conscious sedation would give rapid and predictable sedation of appropriate depth and duration for the procedure, and the doses of sedative agents used should be such that no patient develops airway difficulties or loses verbal contact. In practice conscious sedation using a fixed dose per kilogram of bodyweight regimen is difficult to achieve due to pharmacodynamic and pharmacokinetic variability between individuals. Titration of drugs to effect also requires a high level of skill and an appreciation of the pharmacology of the agents used. In our study, using fixed doses of sedative agents per kilogram of bodyweight, six out of 1,000 cases $(0.6 \%)$ lost verbal contact, either during the procedure or in the early recovery period. Although this is a small percentage and airway control was maintained in all patients, there is potential for airway obstruction and apnoea.

Following the initial 1,000 cases and particularly when the option of general anaesthesia was withdrawn from dental practice, the technique of drug titration against response was adopted to avoid the small percentage of deep sedation that is unacceptable. Logically titrating drugs with different half lives should present difficulties, however in clinical practice titration does work but also avoids that extremely small number of children that proceed to deep sedation unexpectedly.

To arrive at a combination of safe and predictable sedative agents, prior to our 1,000 reported cases, we looked at the effects of differing ketamine doses. We observed that ketamine administered at $0.3-0.4 \mathrm{mg} / \mathrm{kg}$ bodyweight produced the optimum degree of sedation and analgesia. At $0.2 \mathrm{mg} / \mathrm{kg}$, there was insufficient analgesia for the children to tolerate the local anaesthetic injection. At doses approaching the well documented GA dose of $2 \mathrm{mg} / \mathrm{kg}$ the clinical picture becomes different to the trained eye.

Although patients appear awake with their eyes open and even spontaneously moving and maintaining their airway without assistance, their movements are not related to surgical stimulation and are independent from command. At this dose level it is possible to perform an extraction without local anaesthesia.

Several sedation studies use ketamine at doses of greater than one $\mathrm{mg} / \mathrm{kg}$ for paediatric dental sedation. Although we feel at these doses ketamine induces general anaesthesia and goes beyond conscious sedation, is this a relatively safe regimen to use in outpatient dentistry? We feel it is not. Firstly, there is a high risk of airway difficulties because ketamine increases airway secretions ${ }^{8}$ and can increase the sensitivity of airway reflexes at higher doses leading to laryngospasm, and it is well documented that there is a higher risk of airway obstruction during sedation with ketamine than other agents. ${ }^{9}$ Secondly, in dentistry, there is a higher risk of airway obstruction anyway due to the shared airway.

There is the possibility of reducing the initial dose of ketamine to $0.25 \mathrm{mg} / \mathrm{kg}$ and increasing the dose of midazolam to $0.2 \mathrm{mg} / \mathrm{kg}$, to see if this modification could ensure that $100 \%$ of patients maintain verbal contact throughout. The authors feel that this study should be performed in a hospital environment.

In addition to the described regimen of conscious sedation with ketamine, 
alfentanil and midazolam, other pharmacological agents including propofol and remifentanil could have a role in sedation for paediatric patients. Propofol produces a different character of sedation, which is closer to a pure anxiolysis, although in carefully titrated doses, may be a useful alternative for short procedures, such as single deciduous tooth extraction, which is a very short procedure. With low dose propofol the degree of sedation can be altered rapidly and post-operative recovery is quick..$^{10}$ It has been suggested that a technique for single extractions, using low dose propofol and a systemic analgesic such as alfentanil or indeed ketamine, instead of local anaesthetic infiltration, in order to avoid the side effect of mouth numbness in the recovery period should be investigated. Complications associated with mouth numbness could be potentially serious, such as lip and tongue biting which causes distress to the parents and can cause post-traumatic oedema or infection to the area involved. However this technique may lead to a disguised form of general anaesthetic and therefore in our opinion should only be allowed in a hospital setting.

Remifentanil has the potential for use in small, calculated doses. It would be cost effective, give excellent analgesia and a rapid recovery. However, several studies have found that remifentanil produces apnoea at sub-therapeutic doses, ${ }^{11-12}$ and therefore it should only be used cautiously in self-ventilating patients by experienced personnel. A large prospective study is therefore required to further assess its suitability as an agent for conscious sedation.

Points of interest from our case report include the issue of failed cannulation and the incidence of side effects, notably hypoxia, PONV, emergence reactions and visual disturbance. Seven children refused venous cannulation and their dental work had to be rescheduled. There is a question as to whether children who are agitated or needle phobic should receive oral sedation. Oral sedation does have a long latent period and a delayed recovery. The appropriate anxiety management technique for such patients needs to be investigated, especially as some of them may benefit from general anaesthesia.

Hypoxia, defined as pulse oximeter readings lower than 95\% oxygen saturations, was a surprisingly rare event in our case report, occurring in only $0.8 \%$ of cases. There may be several reasons why the anticipated respiratory depression did not occur. Firstly, the stimulatory effect of ketamine may have countered the respiratory depressant effects of alfentanil. Secondly, the dental treatment may have acted as a respiratory stimulant, and lastly, it is possible that hyperventilation occurred as a result of emergence dreams.

Unexplained crying or laughing occurred in several children during the procedures and this may have been due to emergence reactions, a welldocumented phenomenon with the use of ketamine. ${ }^{14}$ However the parental responses to the questionnaire indicated that children were not troubled beyond the immediate recovery period. Visual disturbances occurred in a surprisingly high $21.6 \%$ of patients, and again this may be an effect of the ketamine as visual disturbances are not known side effects with midazolam or alfentanil, however all had settled by the time the children left recovery. It is possible that the euphoric effects of ketamine in the doses specified may be beneficial to any conscious sedation technique, including in adult sedation.

PONV occurred in 5.3\% of patients, a rate that compares favourably with paediatric patients undergoing dental procedures under general anaesthetic. ${ }^{15-16}$ Anti-emetics were administered to 3.1\% of patients, and it can be argued that all patients should receive routine antiemetic prophylaxis.

Conscious sedation for dentistry must only be provided by teams trained and experienced in providing conscious sedation for dentistry. Specific training and experience in the technique used and patient group treated must be demonstrated. Practices where sedation is used must be subject to inspection and regulation. Practices where the standard techniques of inhalation sedation, intravenous or oral benzodiazepines should be inspected as part of the normal practice inspection process. Practices where other techniques are used should be subject to a more rigorous inspection process.

In outpatient dentistry we feel it is possible to use multi-drug intravenous techniques, as long as the definition of conscious sedation is strictly adhered to. If non-anaesthetists are limited to the use of single agent sedation, for example midazolam, predicting the appropriate dose for each child would be very difficult. After giving a fixed dose of sedative agent, if the child did not then tolerate the procedure, further top-ups would be required. By administering further doses before the peak effect of the initial dose had occurred, this would create 'stacking' of doses with possible over-sedation, and over-sedation would be extremely difficult to regulate in clinical practice. We feel it is more acceptable not to limit the number of agents used, but to adhere strictly to 'conscious' sedation. Each patient should be specifically assessed and provided with the most appropriate anxiety management technique for that patient undergoing the specific dental treatment on that day.

\section{CONCLUSION}

The authors feel that in the appropriate circumstances the use of a combination of drugs in an appropriate conscious sedation mixture is an appropriate conscious sedation technique. This is particularly indicated for those patients who do not cope well with sedation provided by the standard techniques or for whom the standard techniques are felt to be inappropriate. The recommendations from the GDC to sedationists should emphasise the level of consciousness required rather than the drug or drugs used to produce the effect.

We would like to thank the following dental surgeries and surgeons for their help in the preparation of this report: Highland View Dental Surgery, Leigh-On-Sea, Essex (Dr Sanjay Chopra, Dr Sarita Chopra, Dr Philip Hayter); Crown Court Clinic, Rochester, Kent (The late Dr Denis Batchelor); St. Mark's Dental Surgery, Cambridge (Dr Magdi Bassilious) Some of the data in this report were presented at the $13^{\text {th }}$ South West Thames Anaesthesia Update Meeting, Winter 2002.

1. A Conscious Decision. A review of the use of general anaesthesia and conscious sedation in primary dental care. Report by a Group chaired by the Chief Medical Officer and Chief Dental Officer. Department of Health, July 2000.

2. Poswillo D. General anaesthesia, sedation and resuscitation in dentistry. Report of an Expert Working Party prepared for the Standing Dental Advisory Committee. (The Poswillo Report.) Department of Health, 1990.

3. Maintaining standards. Guidance to dentists on professional and personal conduct. London: General Dental Council, 1998.

4. Implementing and ensuring safe sedation practice for healthcare procedures in adults. Academy of Medical Royal Colleges and their Faculties: Report of an Intercollegiate Working Party chaired by the Royal College of Anaesthetists, November 2001.

5. Wathen J E, Roback M G, Mackenzie T et al. Does midazolam alter the clinical effects of intravenous 
ketamine sedation in children? A double-blind, randomized controlled, emergency department trial. Ann Emerg Med 2000; 36: 579-588.

6. Conscious sedation. A referral guide for dental practitioners. A Joint Initiative by the Dental Sedation Teachers' Group and the Society for the Advancement of Anaesthesia in Dentistry. Available at http://www.dstg.co.uk.

7. American Society of Anesthesiologists. Continuum of depth of sedation. Definition of general anesthesia and levels of sedation/analgesia. Park Ridge (IL): The Society, 1999

8. Aun C S T. New IV agents. Br J Anaesth 1999; 83: $29-44$.
9. Birkham J, Shamash R, Gutman D. Haifa, Israel. Ketamine - dissociative anaesthesia in paediatric oral surgery. J Oral Surg 1971; 29: 853-857.

10. Rodrigo M R, Jonsson E. Conscious sedation with propofol. Br Dent J 1989; 166: 75-80.

11. Litman R S. Conscious sedation with remifentanil and midazolam during brief painful procedures in children. Arch Pediatr Adolesc Med 1999;

153: 1085-1088.

12. Litman R S. Conscious sedation with remifentanil during painful medical procedures. J Pain Symptom Manage 2000; 19: 468-471.

13. Servin F S, Raeder J C, Merle J C et al. Remifentani sedation compared with propofol during regional anaesthesia. Acta Anaesthesio/ Scand 2002; 46: 309-315

14. Bowdle TA, Radant A D, Cowley D S et al. Psychedelic effects of ketamine in healthy volunteers. Anesthesiology 1998; 88: 82-88.

15. Enever G R, Nunn J H, Sheehan J K. A comparison of post-operative morbidity following outpatient dental care under general anaesthesia in paediatric patients with and without disabilities. Int J Paediatr Dent 2000; 10: 120-125.

16. Apfel C C, Kranke P, Eberhart L H J et al. Comparison of predictive models for postoperative nausea and vomiting. Br J Anaesth 2002; 88: $234-240$. 